\title{
乌s \\ Formation and acceleration of uniformly filled ellipsoidal electron bunches obtained via space-charge-driven expansion from a cesium-telluride photocathode
}

\author{
P. Piot, ${ }^{1,2}$ Y.-E Sun, ${ }^{2}$ T. J. Maxwell, ${ }^{1,2, *}$ J. Ruan, ${ }^{3}$ E. Secchi, ${ }^{2, \dagger}$ and J. C. T. Thangaraj ${ }^{2}$ \\ ${ }^{1}$ Northern Illinois Center for Accelerator \& Detector Development and Department of Physics, \\ Northern Illinois University, DeKalb, Illinois 60115, USA \\ ${ }^{2}$ Accelerator Physics Center, Fermi National Accelerator Laboratory, Batavia, Illinois 60510, USA \\ ${ }^{3}$ Accelerator Division, Fermi National Accelerator Laboratory, Batavia, Illinois 60510, USA
}

(Received 10 October 2012; published 22 January 2013)

\begin{abstract}
We report the experimental generation, acceleration, and characterization of a uniformly filled electron bunch obtained via space-charge-driven expansion (often referred to as "blow-out regime") in an L-band $(1.3-\mathrm{GHz})$ radiofrequency photoinjector. The beam is photoemitted from a cesium-telluride semiconductor photocathode using a short $(<200 \mathrm{fs})$ ultraviolet laser pulse. The produced electron bunches are characterized with conventional diagnostics and the signatures of their ellipsoidal character are observed. We especially demonstrate the production of ellipsoidal bunches with charges up to $\sim 0.5 \mathrm{nC}$ corresponding to a $\sim 20$-fold increase compared to previous experiments with metallic photocathodes.
\end{abstract}

DOI: $10.1103 /$ PhysRevSTAB.16.010102

\section{INTRODUCTION}

Three-dimensional uniformly filled ellipsoidal charge distributions produce space-charge fields that have a linear dependence on position within the distribution $[1,2]$. The resulting density distributions are therefore immune to space-charge-induced phase space dilution. Besides mitigating emittance dilution, these ellipsoidal bunches are notably less prone to halo formation thereby making these distributions attractive for, e.g., high-average-power freeelectron lasers (FELs) [3].

Several schemes to generate uniformly filled ellipsoidal distribution via photoemission have been proposed over the recent years. One class of method relies on the precise temporal shaping of the photocathode drive-laser pulse using either a pulse-stacking technique [4] or introducing and controlling spatiotemporal aberrations [5]. An alternative scheme, which recently regained interest, was proposed by Serafini [6] and subsequently refined by Luiten et al. [7]. The latter proposal uses an ultrashort laser impinging on a prompt photoemitter subject to a strong accelerating electric field $E_{\text {acc }}$. The operating parameters of the electron source are chosen such that the distribution evolution is dominated by linear space-charge force. This space-charge-dominated expansion, also referred to as the "blow-out regime" [6], is achieved provided the condition [7]

\footnotetext{
*Current address: Stanford Linear Accelerator Center, Menlo Park, CA 94025, USA.

${ }^{\dagger}$ Permanent address: Politecnico di Milano, 20156 Milano, Italy.

Published by the American Physical Society under the terms of the Creative Commons Attribution 3.0 License. Further distribution of this work must maintain attribution to the author(s) and the published article's title, journal citation, and DOI.
}

PACS numbers: 29.27.-a, 41.85.-p, 41.75.Fr

$$
\frac{e E_{\mathrm{acc}} c \tau_{l}}{m c^{2}} \ll \frac{\sigma_{0}}{\epsilon_{0} E_{\mathrm{acc}}} \ll 1
$$

is fulfilled. Here $\tau_{l}, c, \epsilon_{0}, m$, and $e$ are respectively the duration of the photoemission process, the speed of light, the electric permittivity of vacuum, and the electronic mass and charge. The parameter $\sigma_{0} \equiv Q /\left(\pi r^{2}\right)$ is the initial surface charge density $(Q$ is the bunch charge and $r$ the radius of the laser transverse distribution on the photocathode). The conditions given by Eq. (1) lead to constraints in the parameter space $\left(\sigma_{0}, E_{\text {acc }}\right)$ that would result in a ellipsoidal bunch.

The Serafini-Luiten technique is relatively simple to implement, especially with the availability of a short-pulse laser, but has limitations regarding the achievable minimum transverse emittance [8]. This is because for a given accelerating field at the photocathode and desired bunch charge, the blow-out regime requires large transverse laser spots at the photocathode with consequent large thermal transverse normalized emittance. Therefore this type of operating regime has generally not been selected for, e.g., FEL applications. The technique has however become popular for use in the ultrafast electron diffraction experiment where ultrashort low-charge electron bunches are generally needed $[9,10]$.

We consider a L-band radiofrequency ( $\mathrm{rf}$ ) gun with a typical peak accelerating field of $E_{0}=35 \mathrm{MV} / \mathrm{m}$ corresponding to an accelerating field $E_{\text {acc }}=E_{0} \sin \left(\varphi_{l}\right)$ at the photocathode surface (where $\varphi_{l}$ is the injection phase referenced to the zero-crossing point). The conditions for ellipsoidal-bunch generation using the blow-out regime can in principle be realized with $\tau_{l}=50 \mathrm{fs}$; see Fig. 1(a). Figure 1 visualizes the ellipsoidal-bunch existence domain in the $\left(\sigma_{0}, E_{\text {acc }}\right)$ parameter space. The dark area corresponds to a region where none or only one of the conditions 

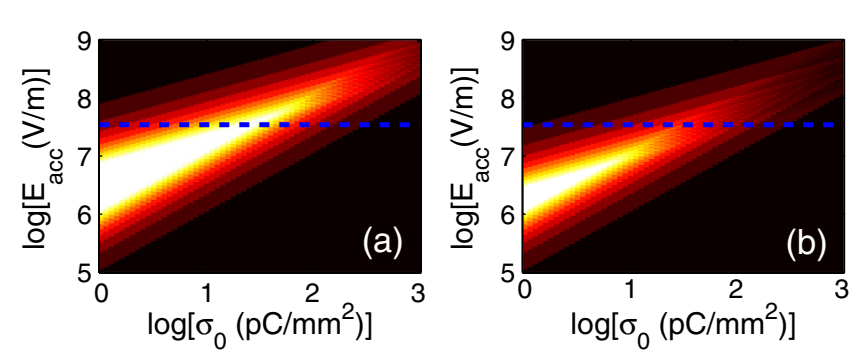

FIG. 1. Domain of existence of the blow-out regime (lighter colors) in the $\left(\sigma_{0}, E_{\mathrm{acc}}\right)$ parameter space for $\tau_{l}=50 \mathrm{fs}$ (a) and $\tau_{l}=200 \mathrm{fs}(\mathrm{b})$. The horizontal blue dashed lines correspond to $E_{0}=35 \mathrm{MV} / \mathrm{m}$. (This figure was adapted from Ref. [13].)

given in Eq. (1) is satisfied while the darker orange area indicates the case of equalities in Eq. (1). The lighter colors in Fig. 1 delineate areas where the conditions in Eq. (1) are fulfilled. A longer pulse length $\tau_{l}=200 \mathrm{fs}$ can still support the scheme; see Fig. 1(b). Further increasing $\tau_{l}$ would require lower values of $E_{0}$ and $\sigma_{0}$ to meet the blow-out regime conditions [Eq. (1)] resulting in operating points that might not be relevant to applications demanding significant (sub-nC) charge per bunch, e.g., high-averagepower FELs [11,12].

For prompt-emission photocathodes, $\tau_{l}$ is comparable to the laser-pulse duration and the formation of ellipsoidal bunches was experimentally confirmed [13-15]. For semiconductor high-quantum-efficiency photocathodes, the slower photoemission response might affect the production of ellipsoidal bunches via the blow-out regime as pointed out in Ref. [16].

To date, there has been no conclusive investigation regarding the compatibility of the blow-out regime with semiconductor photocathodes $[17,18]$. Such an investigation is the prime purpose of this paper. In addition, the presented experimental results are obtained in an L-band photoinjector with $E_{0} \simeq 35 \mathrm{MV} / \mathrm{m}$, whereas previous successful experiments have been carried out at S-band photoinjectors with higher values of typically $E_{0} \sim 100 \mathrm{MV} / \mathrm{m}$ $[13,14]$. The present work supports the use of the blow-out regime in low-frequency electron guns with a limited peak field. Such guns are foreseen as sources for high-frequency multiuser FEL facilities [19,20].

\section{THE AO PHOTOINJECTOR SETUP}

The numerical and experimental investigations of ellipsoidal-bunch production from a semiconductor photocathode and their subsequent acceleration to $\sim 16 \mathrm{MeV}$ were carried out at the now-decommissioned Fermilab's A0 photoinjector; see Fig. 2 [21]. In brief, electron bunches are generated via photoemission from a cesium-telluride $\left(\mathrm{Cs}_{2} \mathrm{Te}\right)$ photocathode $[22,23]$ located on the back plate of a $1.3-\mathrm{GHz} 1+1 / 2$ cell $\mathrm{rf}$ gun. The laser pulse impinges the photocathode under normal incidence. The rf gun is surrounded by three solenoidal lenses that control the

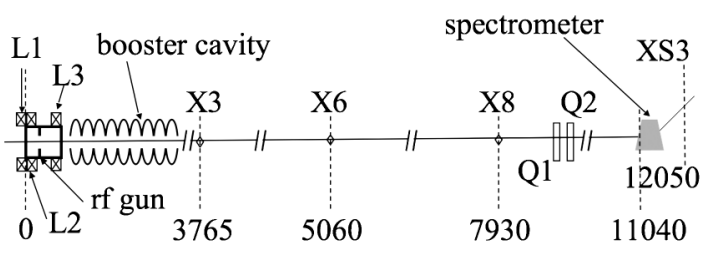

FIG. 2. Top view of the A0PI setup displaying elements pertinent to the present simulations and experiments. The " $L$ " refers to solenoidal lenses, " $\mathrm{X}$ " to diagnostic stations (beam viewers and/or multislit masks location), and " $Q$ " to quadrupole magnets. The distances are in $\mathrm{mm}$ and referenced to the photocathode surface.

beam's transverse size and emittance. The beam is then accelerated in a $1.3 \mathrm{GHz}$ superconducting rf cavity [24]. Downstream of the booster cavity, the beam line includes quadrupoles and steering dipole magnets, and diagnostics stations. The transverse density diagnostics are based on cerium-doped yttrium aluminum garnet (Ce:YAG) screens (labeled as " $\mathrm{X}$ " in Fig. 2). A multislit mask insertable at $\mathrm{X} 3$ can be used to measure the transverse emittance by analyzing the resulting beamlet's distribution at the X6 screen [25]. The multislit mask consists of $50 \mu \mathrm{m}$-wide slits made out of a 3-mm-thick tungsten plate. The slits are separated by $1 \mathrm{~mm}$. Finally, at its end, the beam line incorporates a horizontally bending spectrometer equipped with a Ce:YAG screen (XS3) for energy measurement. The horizontal dispersion value at the XS3 location is $|\eta|=$ $317 \mathrm{~mm}$. The nominal operating parameters of the A0PI subsystems are gathered in Table I.

Nominally, the A0PI's photocathode laser consists of a frequency-quadrupled neodymium-doped yttrium lithium fluoride (Nd:YLF) laser [26]. Because of its narrow bandwidth $(\Delta \lambda \sim 5 \AA)$, this laser system cannot produce laser pulses with duration $<3$ ps. Therefore a short-pulse laser based on a titanium-sapphire oscillator and regenerative

TABLE I. Nominal settings for the rf gun, booster cavity, and the photocathode UV laser.

\begin{tabular}{|c|c|c|}
\hline Parameter & Value & Units \\
\hline Laser injection phase $\mathrm{a}^{\mathrm{a}}$ & $45 \pm 5$ & rf deg \\
\hline Laser radius on cathode & {$[0.3,2]$} & $\mathrm{mm}$ \\
\hline Laser rms pulse duration & $<200$ & fs \\
\hline Bunch charge & {$[100-700]$} & $\mathrm{pC}$ \\
\hline$E_{z}$ on cathode $\left(E_{0}\right)$ & $33.7 \pm 0.2$ & $\mathrm{MV} / \mathrm{m}$ \\
\hline peak $B_{z}{ }^{\mathrm{b}}(\mathrm{L} 2, \mathrm{~L} 3)$ & $(0.158-0.041)$ & $\mathrm{T}$ \\
\hline Booster cavity gradient & $\sim 12.0$ & $\mathrm{MV} / \mathrm{m}$ \\
\hline Booster cavity phase $^{c}$ & {$[-60-60]$} & rf deg \\
\hline
\end{tabular}

${ }^{\mathrm{a}}$ The phase is referenced with respect to (w.r.t.) the zero-crossing phase.

${ }^{\mathrm{b}}$ The peak field of solenoid L1 was tuned to zero the axial magnetic field on the photocathode.

${ }^{\mathrm{c}}$ The phase is referenced w.r.t. the maximum energy. 

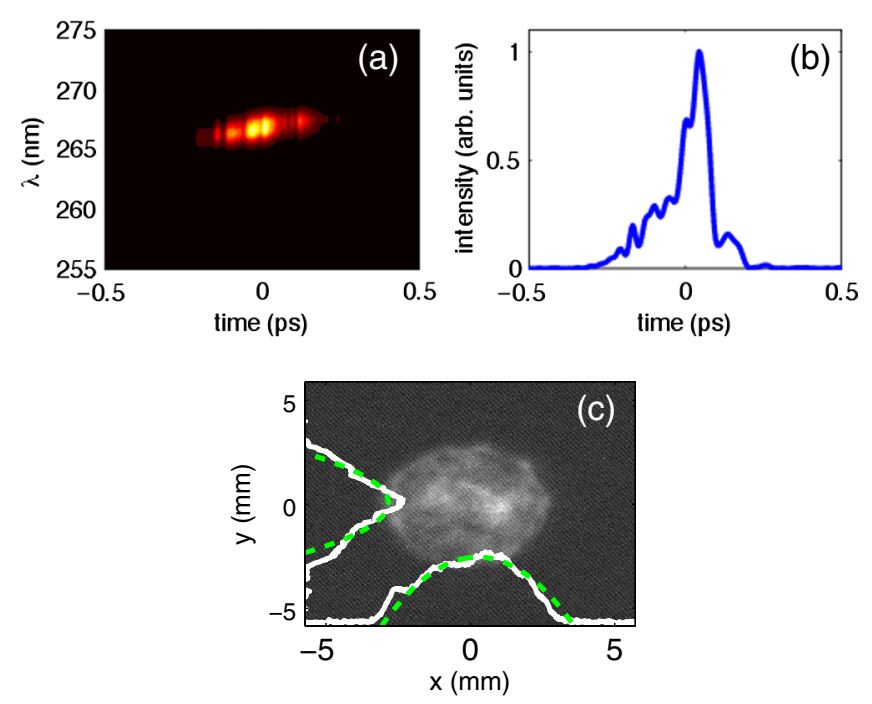

FIG. 3. Characterization of the UV laser pulse. Measured spectrogram (a) using a polarization-gate implementation of the frequency-resolved optical gating (FROG) technique and reconstructed temporal distribution (b) of the UV pulse (the inferred rms UV pulse duration is $122 \mathrm{fs}$ ). Typical transverse spatial distribution of the UV laser pulse on the photocathode (c) with associated projection (white traces) and parabolic fits (green traces).

amplifier was recently installed [27]. The system also includes an acousto-optic programmable dispersive filter system to control the laser shape [28,29]. The generated $3-\mathrm{mJ}$ infrared pulses $(\lambda=800 \mathrm{~nm})$ with duration of $\sim 50 \mathrm{fs}$ (rms) are frequency tripled using a two-stage frequency up-conversion scheme [30]. The second-harmonic-generation (SHG) and sum-frequency-generation (SFG) stages consist of $\beta$-barium borate $(\beta$-BBO) crystals of respective thicknesses 300 and $150 \mu \mathrm{m}$. The frequency up-conversion was optimized to preserve the short pulse duration: the thicknesses of the $\beta$-BBO crystals were selected based on numerical simulations using a customized version of SNLO [31], and a calcite crystal was included to compensate for delay accumulation as the 800-nm and SHG-generated 400-nm pulses copropagate in the 800-nm half-wave plate needed to match the polarizations prior to the SFG stage. The resulting ultraviolet (UV) pulses have a root-mean-square (rms) duration of $\sim 150$ fs as measured using a polarization-gate version $[32,33]$ of a frequency-resolved optical gating (FROG) method [34,35]; see Fig. 3. The estimated duration of the UV pulse is below $\sim 200 \mathrm{fs}$ at the photocathode (after transport through a $\sim 20$-m long optical beam line and passage through three vacuum windows). Figure 3 also shows the typical transverse density of the UV laser system as measured at the "virtual cathode," a UV-sensitive screen located outside of the vacuum chamber, providing a one-to-one optical image of the laser on the photocathode.

\section{NUMERICAL SIMULATIONS}

To confirm whether the A0PI facility could support the production of ellipsoidal bunches using the blow-out regime, particle-in-cell simulations were performed using the program ASTRA [36] which includes a quasistatic space-charge algorithm.

The initial conditions for the electron-beam generation are dictated by the photocathode drive laser. For the present simulation, the UV laser pulse was taken to follow a Gaussian distribution with 200-fs (rms) duration. However, contrary to metallic photocathodes, their semiconductor counterparts have a finite emission time generally described as a diffusion process. Measurements performed for the negative-electron affinity cathode have validated this model and response times in the ps regime were reported $[37,38]$. To our knowledge there is no corresponding experimental data for $\mathrm{Cs}_{2} \mathrm{Te}$ cathodes and to date the response times were only investigated numerically via Monte Carlo simulations [39] of the three-step model [40]. These numerical results indicate a significant spread in electron transit time for an initial Dirac-like laser excitation [39]; see Fig. 4(a). In particular, it is found that $90 \%$ of the electrons are emitted within a temporal window of $370 \mathrm{fs}$. The distribution also has a trailing electron population that extents to $\sim 1$ ps after the laser impinged the photocathode. In order to take into account the finite emission time in our simulation, we parametrized the data shown in Fig. 4(a) with the function

$$
\Lambda(t)=a_{1} e^{-t / \tau_{1}}+a_{2} e^{-t / \tau_{2}}
$$
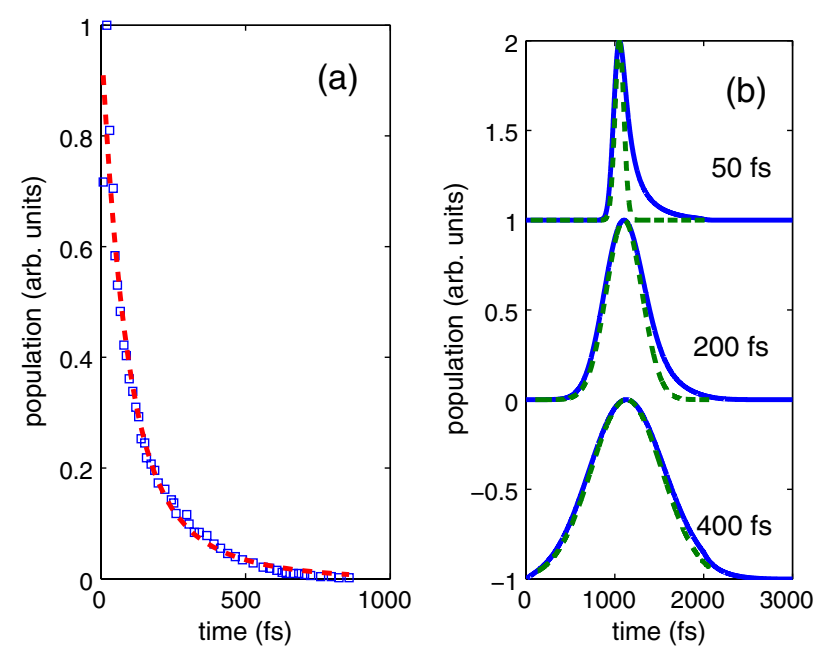

FIG. 4. Monte Carlo simulation (squares) taken from Ref. [39] and parametrization (dashed line) of the electron transit time from an initial Dirac-like laser excitation (a). Impact of the slow response time on the photoemission profile (blue traces) for three different Gaussian-like laser profiles (green dashed traces) of indicated rms duration (b). The traces in plot (b) are vertically offset for clarity. Larger time ordinates correspond to the bunch tail. 
where $a_{i}$ and $\tau_{i}$ are fitting parameters. Note that the motivation of this mathematical parametrization is to provide an analytic function for simulation purposes rather than to attempt a description of the physics associated with the response profile. The temporal charge distribution during the photoemission process (at the photocathode surface) is then taken as the convolution

$$
Q(t)=\int_{-\infty}^{+\infty} Q_{0}\left(t^{\prime}\right) \Lambda\left(t-t^{\prime}\right) d t^{\prime}
$$

where $Q_{0}(t)$ is a Gaussian charge distribution with rms duration given by the drive-laser pulse duration $\sigma_{t}$. The results of such a convolution appear in Fig. 4(b) for different laser profiles. The latter figure shows that for laser pulses with $\sigma_{t} \leq 400 \mathrm{fs}$, the finite emission time of $\mathrm{Cs}_{2} \mathrm{Te}$ significantly alters the charge emission profile and especially leads to asymmetric emission profiles with long trailing tails. In order to explore the effects associated with the finite response time of the $\mathrm{Cs}_{2} \mathrm{Te}$ cathodes, the photoemission process was simulated using the emission profile described by Eq. (3). The transverse distribution of the laser was taken as a $2 \sigma$-clipped Gaussian distribution.

An example of simulated spatiotemporal $(z, x)$ densities and associated longitudinal charge distributions appears in Fig. 5 for a charge of $Q=250 \mathrm{pC}$. The simulations confirm that for low-charge bunches, the A0PI can operate in the blow-out regime. Increasing the charge to higher values (up to $1000 \mathrm{pC}$ ) does not significantly alter the ellipsoidal character; see Fig. 6 . For the range of charge attainable at the A0PI, the space-charge-driven bunch length expansion is strongly suppressed once the bunch has been accelerated by the booster cavity; see Fig. 6(g).
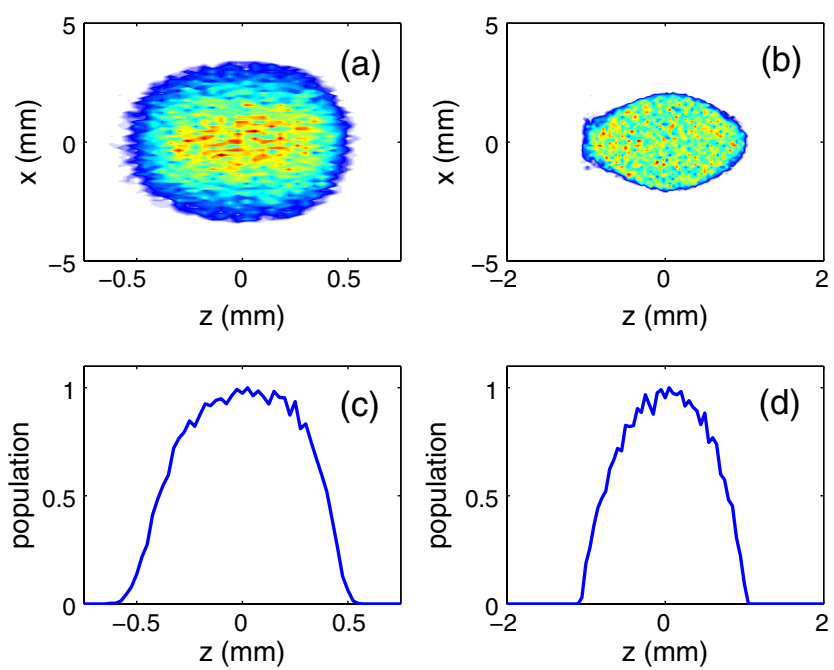

FIG. 5. Simulated spatiotemporal distribution $(z, x)$ at $s=$ $0.47 \mathrm{~m}$ (a) and $s=3.77 \mathrm{~m}$ (b) (corresponding to X3 screen) w.r.t. to the photocathode and associated longitudinal charge distributions [(c) and (d), respectively]. The charge is $250 \mathrm{pC}$ and the rms laser transverse size is $\sigma_{c}=1 \mathrm{~mm}$. In these plots the tail of the bunch is at $z>0$.
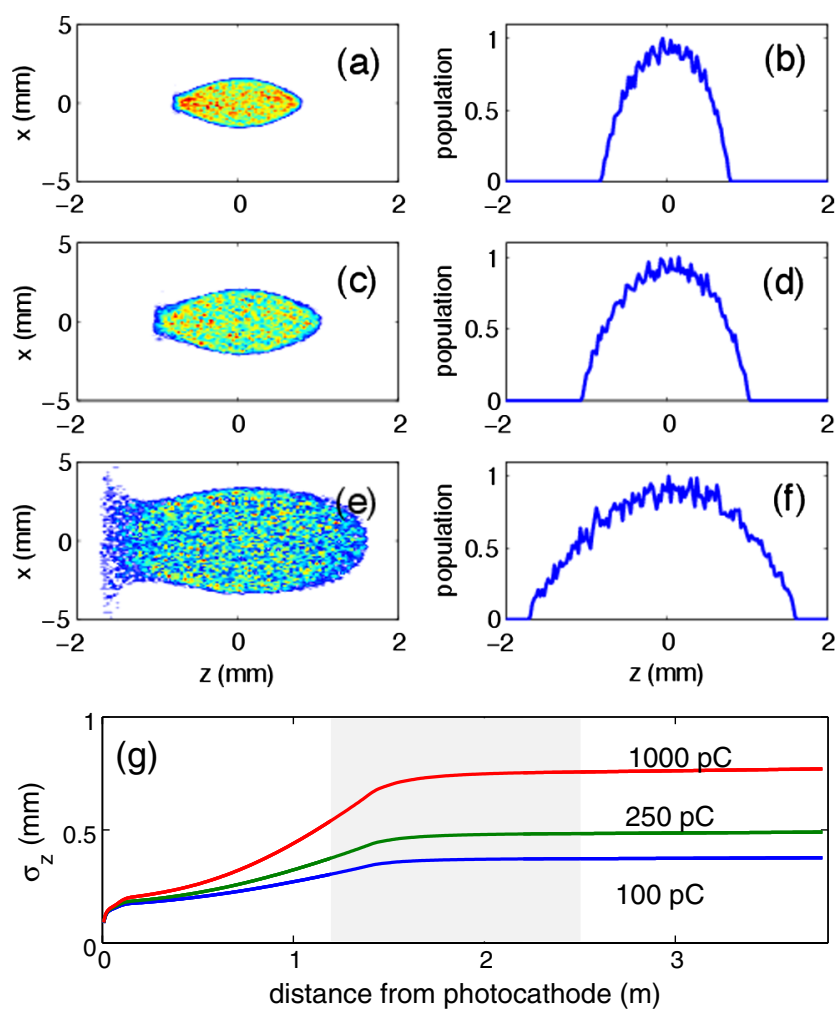

FIG. 6. Simulated spatiotemporal distribution $(z, x)$ (left column) and associated longitudinal charge distribution (right column) simulated at X3 $(s=3.77 \mathrm{~m})$ for three cases of charges 100 [(a), (b)], 250 [(c), (d)], and 1000 pC [(e), (f)]. In these plots the tail of the bunch is at $z>0$. Plot (g) illustrates the rms bunch length evolution along the beam line up to $z=3.77 \mathrm{~m}$ (location of X3) for the three cases of bunch charge. The shaded area in plot $(\mathrm{g})$ indicates the location of the booster cavity. The rms laser transverse size is kept constant to $\sigma_{c}=1 \mathrm{~mm}$ for the three cases of charges.

To investigate the domain of existence of the blow-out regime in the $\left(\sigma_{0}, E_{0}\right)$ parameter space using the realistic beam line and external fields of the A0 photoinjector, we introduce a figure of merit to quantify the ellipsoidal character of the bunch distribution. Since one of the properties associated with such a distribution is the linearity of the two-dimensional subphase spaces, we introduce the mean Euclidean distance associated with the $\left(u, u^{\prime}\right)$ trace space as

$$
\mathcal{D}_{u} \equiv \frac{1}{N} \sum_{i=1}^{N} d_{u, i}, \quad \text { with } d_{u, i} \equiv \frac{\left|u_{i}^{\prime}-m_{u} u_{i}-q_{u}\right|}{\sqrt{1+m_{u}^{2}}}
$$

where $u \in[x, y, z]$ and the summation is performed over the number of macroparticles representing the bunch, $\left(u_{i}, u_{i}^{\prime}\right)$ are the trace-space coordinates of the $i$ th macroparticle, and $m_{u}$ and $q_{u}$ are respectively the slope and intersect of the linear regression of the macroparticle distribution in $\left(u, u^{\prime}\right)$. In Ref. [14], a different figure of merit 
based on the temporal asymmetry was introduced but is not as convenient to automatically compute compared to the $\mathcal{D}_{u}$. Nonetheless we verified that the two metrics were consistent. For the nominal gun field $E_{0}=35 \mathrm{MV} / \mathrm{m}$, the charge and transverse size of the laser were varied. For all the simulations, the photoemission temporal profile shown in Fig. 4(b) ["200 fs" case] was used. The results, summarized in Fig. 7, confirm that despite the low-field L-band rf gun and the long emission time, the configuration can operate in the blow-out regime over a large range of charge. The metric $\mathcal{D}_{z}$ was chosen as it quantifies the linearity of the longitudinal phase space. We find that for each charge $\mathcal{D}_{z}$ is minimized for a slightly different value of $\sigma_{0}$. The locus of minima corresponds to the condition for forming an optimum ellipsoidal bunch [with spatiotemporal distribution shown in Fig. 7(c)]. For larger values of $\sigma_{0}$, the image charge prevails and confers the observed asymmetry egg-shaped spatiotemporal distribution [exemplified in Fig. 7(d)]. Finally for low $\sigma_{0}$ the required beam size becomes large and leads to the alteration of the ellipsoidal character due to nonlinearity of the off-axis fields and nonlinear correlation between the transverse coordinates and time [as investigated in Ref. [41] and shown in Fig. 7(b)]. We also note that $\mathcal{D}_{z}$ increases with the bunch charge so that low charges are more favorable to the blow-out regime for our gun configuration-this is where most of the experimental work as been carried to date [14].

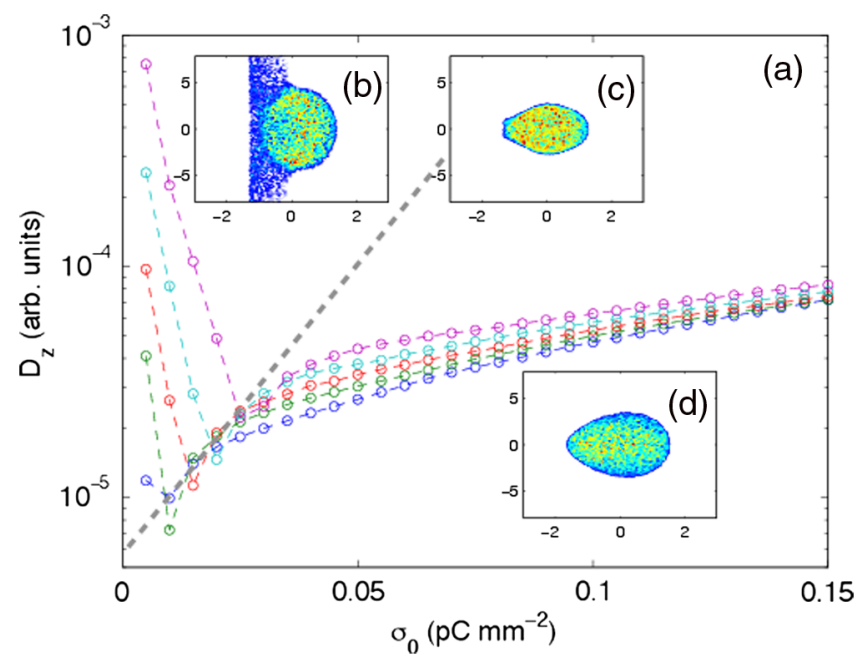

FIG. 7. Computed $\mathcal{D}_{z}$ metric [see Eq. (4)] (a) for bunch charges of 100, 250, 500,1000, and $2000 \mathrm{pC}$ (shown respectively as blue, green, red, cyan, and magenta symbols) as a function of cathode surface density $\sigma_{0}$. The gray line indicates loci of minimum $\mathcal{D}_{z}$ and the insets (b), (c), and (d) show typical spatiotemporal distributions simulated for the three regimes delineated by the gray line; see text for details. The simulation results are evaluated at the location of X3 in Fig. 2. The insets plot horizontal (respectively vertical) axes corresponding to $z$ (respectively $y$ ) both in units of $\mathrm{mm}$.

\section{EXPERIMENTAL RESULTS AND ANALYSIS}

Several experiments were performed to explore the operation of the A0PI in the blow-out regime. These included the measurements of the spatiotemporal $(z \propto t, y)$ distributions and associated current distribution, the estimate of longitudinal-phase-space (LPS) chirp. Finally, we also measured the transverse emittances. When possible the measurements were performed for different charge and laser spot size on the photocathode.

\section{A. Transverse beam density}

Measuring the transverse beam density provided a first hint of the ellipsoidal character of the bunch distribution as depicted in Fig. 8(a). The observed transverse distribution has projections that follow a parabolic distribution and comprise a sharp edge as expected. Slight deviations from the expected projection are due to nonuniformities but do not appear to significantly alter the blow-out regime. For comparison, Fig. 8(b) displays a typical distribution measured at the same location when the bunch is photoemitted using the 3-ps long Nd:YLF laser. Varying the charge density was also found to significantly spoil the parabolic profiles.

\section{B. Longitudinal-phase-space chirp}

A second set of experiments consisted in measuring the incoming longitudinal-phase-space (LPS) chirp. Given the initial LPS coordinates of an electron $\left(z_{0}, \delta_{0}\right)$, the relative momentum offset downstream of the booster cavity operated with an off-crest phase $\varphi$ and accelerating voltage $V$ is

$$
\delta=\frac{\bar{E}_{0}}{\bar{E}} \delta_{0}-\frac{e V k \sin \varphi}{\bar{E}} z_{0}
$$

where $\bar{E} \equiv \bar{E}_{0}+e V \cos \varphi$ with $\bar{E}_{0}$ and $e$ being respectively the initial beam's mean energy and electronic charge and $k \equiv 2 \pi / \lambda$ (where $\lambda \simeq 0.23 \mathrm{~cm}$ is the wavelength associated to the fundamental mode of the booster cavity). The
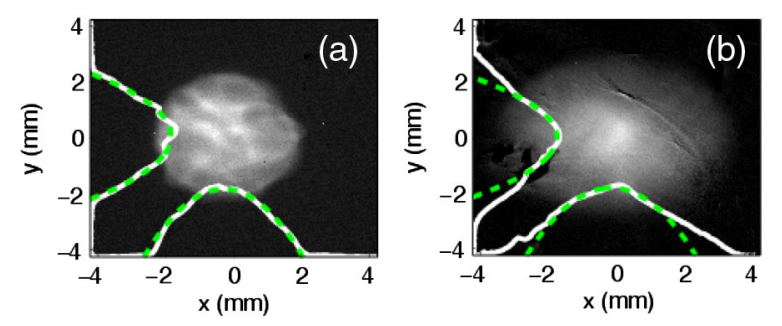

FIG. 8. Examples of measured spatial $(x, y)$ distribution and associated projections (white solid lines) with parabolic fit (green dash lines). Images (a) and (b) respectively correspond to the beam being photoemitted using the ultrashort Ti:Sp and the picosecond Nd:YLF laser systems. The beam density is measured at the Ce:YAG screen X6 (see Fig. 2) for $Q \simeq 250 \pm$ $50 \mathrm{pC}$. 
latter equation is valid provided $k \sigma_{z, 0} \ll 1$ where $\sigma_{z, 0}$ is the incoming bunch length.

Assuming the bunch to be relativistic so that $z=z_{0}$, the final LPS correlation downstream of the booster cavity is

$$
\langle z \delta\rangle=\left\langle z_{0} \delta_{0}\right\rangle \frac{\bar{E}_{0}}{\bar{E}}-\frac{e V}{\bar{E}} k\left\langle z_{0}^{2}\right\rangle \sin \varphi
$$

where $\left\langle z_{0} \delta_{0}\right\rangle$ is the initial LPS correlation and $\left\langle z_{0}^{2}\right\rangle \equiv \sigma_{z, 0}^{2}$ is the squared rms bunch length (the $\langle u\rangle$ notation indicates the statistical averaging of the variable $u$ over the LPS distribution). Setting $\varphi=\varphi_{0}$, where $\varphi_{0}$ is the phase resulting in an upright LPS downstream of the booster cavity $(\langle z \delta\rangle=0)$, provides the incoming LPS chirp

$$
\mathcal{C} \equiv \frac{\left\langle z_{0} \delta_{0}\right\rangle}{\left\langle z_{0}^{2}\right\rangle}=\frac{e V}{\bar{E}_{0}} k \sin \varphi_{0}
$$

Experimentally, $\varphi_{0}$ can be inferred by minimizing the fractional momentum spread as measured at XS3 as illustrated in Figs. 9(a)-9(c). In principle, the technique could
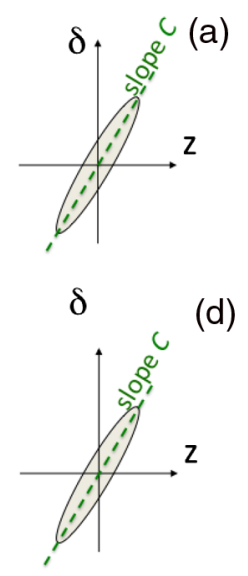

(d)
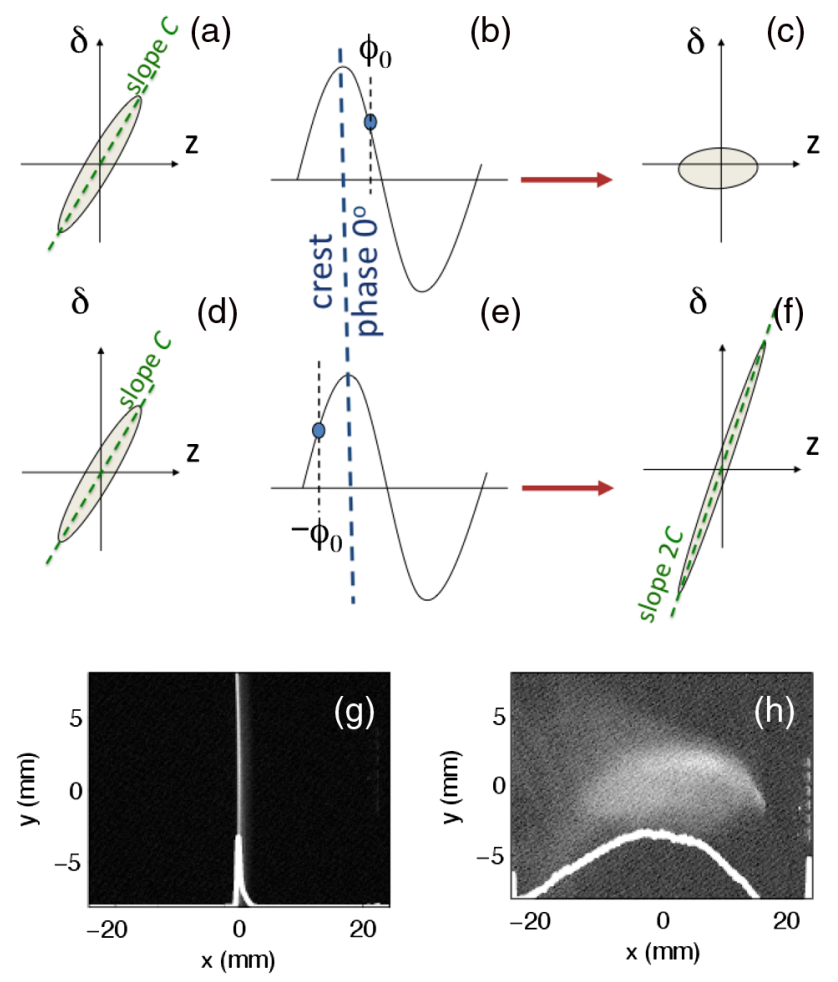

FIG. 9. Principle of measurement of the LPS chirp [(a), (b), and (c)] and bunch length [(d), (e), and (f)] measurements. The left [plots (a), (d)], middle [plots (b), (e)], and right [plots (c), (f)] columns show respectively the initial LPS, the location of the bunch (shown as a blue circle) w.r.t. the accelerating voltage, and the final LPS. The images $(\mathrm{g})$ and $(\mathrm{h})$ respectively show an example distribution measured at the XS3 location for minimum-energy-spread and off-crest phase settings; see text for details. In these latter images the horizontal axis corresponds to the energy-dispersed direction $[x[\mathrm{~mm}] \simeq 317 \delta$ where $\delta$ is the unitless fractional momentum spread]. also allow for the measurement of the uncorrelated momentum spread. Unfortunately, the resolution of our imaging system was not adequate to support such a measurement: as shown in Fig. 9(g), the horizontal width of the distribution is extremely small at the limit of our resolution. Given the operating parameters of the rf gun and booster cavity (respectively $\bar{E}_{0}$ and $V$ ), the estimated values of $\mathcal{C}$ for different charge density are summarized in Fig. 10. As discussed in Ref. [42] and observed in Ref. [15], the chirp is not strongly dependent on the charge in the blow-out regime.

As expected for the blow-out regime, the typical offcrest phase required to minimize the final momentum spread is $\sim 55^{\circ}$ which is significantly larger than the phase required for the longer pulses produced with the nominal $\mathrm{Nd}$ :YLF laser $\left(\sim 25^{\circ}\right)$. Such a large off-crest phase is indicative of the strongly correlated longitudinal phase space, i.e., expected from a space-charge-driven expansion. The large correlated energy spread does however not provide information on the quality of the ellipsoidalbunch distribution. The latest can only be assessed by examining the beam distribution and its projections.

\section{Spatiotemporal and current distributions}

In order to measure the spatiotemporal distribution and confirm that the associated current profile is described by a parabolic function, we use a variant of the zero-phasing technique $[43,44]$. The method consists in imparting a chirp on the bunch by operating the booster cavity off

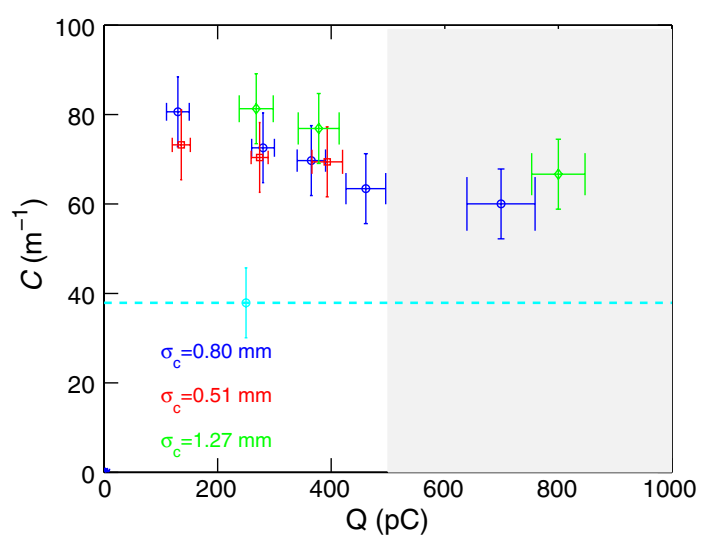

FIG. 10. Measured longitudinal-phase-space chirp $\mathcal{C} \equiv$ $\left\langle z_{0} \delta_{0}\right\rangle /\left\langle z_{0}^{2}\right\rangle$ as a function of charge for three cases of laser transverse rms size $\sigma_{c}$ on the photocathode surface. The horizontal dashed line represents the nominal chirp when the 3-pslong Nd:YLF laser is used for photoemission. The shaded area represents the domain where the parabolic character of the temporal distribution was observed to be distorted and is consequently associated to a regime where a uniformly filledellipsoid bunch is not realized. The chirp associated to the electron bunch produced with the Nd:YLF laser was found to be independent of the charge and only one measurement (performed for $Q=250 \mathrm{pC}$ ) is shown. 
crest. The beam is then horizontally dispersed in a spectrometer. The final horizontal position of an electron at the screen XS3 can be related to the $\left(x, x^{\prime}, \delta\right)$ coordinates upstream of the booster cavity as

$$
x_{f}=R_{11} x+R_{12} x^{\prime}+\eta \delta,
$$

where the $R_{i j}$ 's stand for the transfer matrix elements from the booster cavity exit to XS3 and $\eta$ is the value of the dispersion function at the location of XS3. The fractional momentum offset can be explicited as a function of the longitudinal-phase-space coordinate $\left(z_{0}, \delta_{0}\right)$ upstream of the booster cavity as

$$
x_{f}=R_{11} x+R_{12} x^{\prime}+\eta\left(\frac{\bar{E}_{0}}{\bar{E}} \delta_{0}-\frac{e V k \sin \varphi}{\bar{E}} z_{0}\right) .
$$

Further writing the initial fractional momentum spread as $\delta_{0}=\tilde{\delta}_{0}+\mathcal{C} z_{0}$ where $\tilde{\delta}_{0} \ll \mathcal{C} z_{0}$ is the uncorrelated contribution and taking $\varphi=-\varphi_{0}$ in Eq. (9) yields

$$
x_{f}=R_{11} x+R_{12} x^{\prime}+\eta \tilde{\delta}_{0}+2 \eta \mathcal{C} z_{0} .
$$

By properly tuning the quadrupole magnets $\mathrm{Q} 1$ and Q2, the horizontal position at XS3 is dominated by the last term in Eq. (10), i.e., $x_{f} \simeq 2 \eta \mathcal{C} z_{0}$ (see illustration in Fig. 9) and the transverse $\left(x_{f}, y_{f}\right)$ distribution is representative of the $\left[z_{0}, y_{f}\left(y_{0}\right)\right]$ spatiotemporal distribution. A beam-based calibration procedure was used to infer correspondence to the temporal coordinate by varying the booster cavity phase and recording the beam's centroid motion at XS3. The typical calibration was 24 pixels/ps. An example of measured spatiotemporal distribution appears in Fig. 11. At low charges the current profiles follow the expected parabolic distribution while the higher charge case starts deviating from the parabolic distribution due to distortion appearing in the tail region. This distortion stems from the
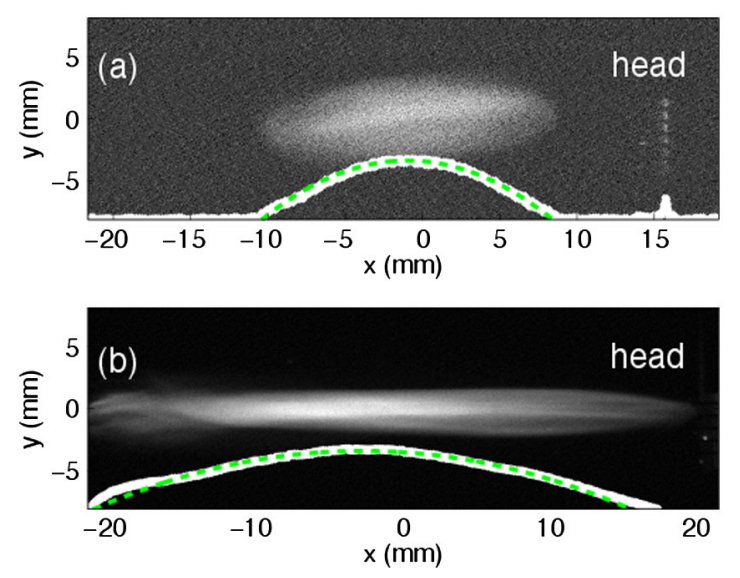

FIG. 11. Measured spatiotemporal $(x \propto t, y)$ distribution and associated projections (white solid lines) with parabolic fit (green dash lines). The beam density is measured at the Ce:YAG screen XS3 (see Fig. 2) for $Q=100 \pm 20 \mathrm{pC}$ (a) and $Q=480 \pm 30 \mathrm{pC}(\mathrm{b})$.

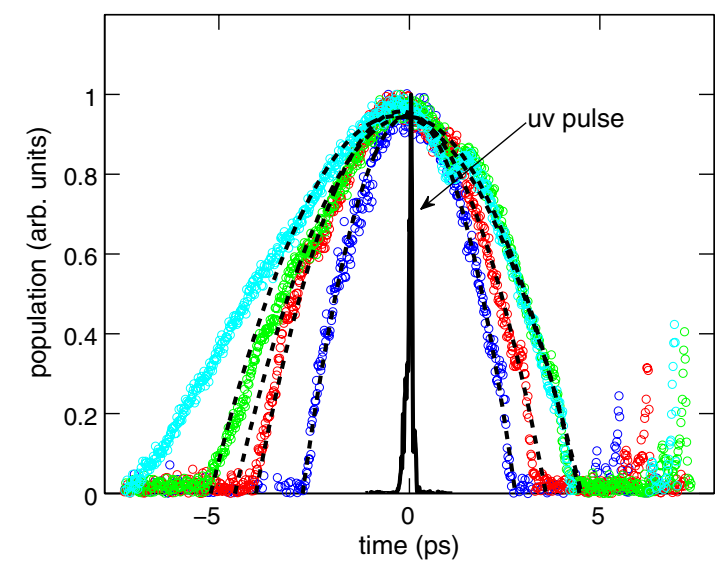

FIG. 12. Measured peak-normalized temporal profiles for $Q \simeq$ 130, 280, 460, and $700 \mathrm{pC}$ (respectively shown as blue, red, green, and cyan circles), compared with the measured uv laser temporal profile (black solid trace). The dashed lines represent the results of parabolic fits to the measured profiles. The head of the bunch corresponds to $t>0$.

image charge becoming more important as the charge increases as previously discussed. The nonuniform features observed on these spatiotemporal distributions are attributed to the initial inhomogeneity of the laser-pulse transverse distribution visible in Fig. 3(c).

Depending on the laser initial spot size, the measured temporal projection follows a parabolic distribution (thereby confirming the ellipsoidal character of the bunch distribution) for charge up to $500 \mathrm{pC}$; see Fig. 12. For the lowest measured charge $(Q=130 \mathrm{pC})$, the measured bunch full-width duration is $\sim 7 \mathrm{ps}$ [corresponding to $\sim 7 /(2 \sqrt{5}) \simeq 1.6 \mathrm{ps}(\mathrm{rms})]$. This final bunch duration represents a $\sim 10$-fold increase compared to the initial laser-pulse rms duration. Larger expansion ratios are observed for higher-charge bunches. Such a large expansion

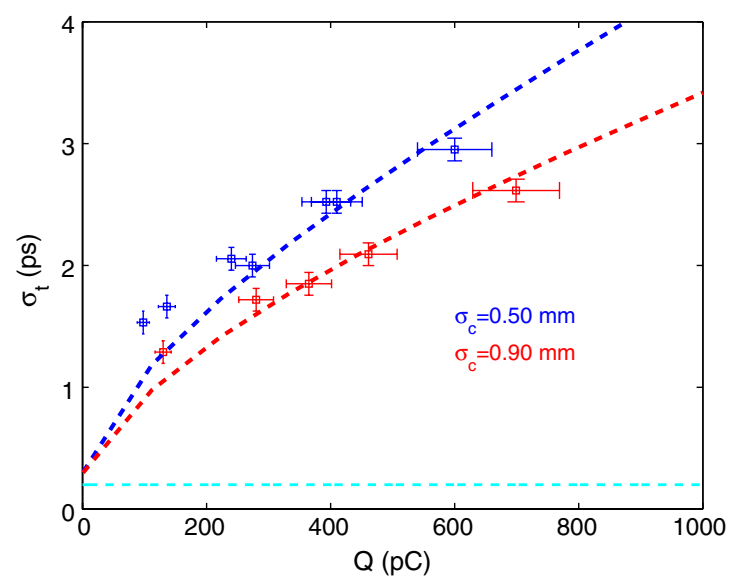

FIG. 13. Measured rms electron-bunch duration (symbols) as a function of charge for two cases of laser transverse spot size $\sigma_{c}$ on the photocathode. The dashed red and blue lines are the corresponding simulations carried with ASTRA. The dashed cyan line represent the UV laser pulse duration. 
is a salient feature of the blow-out regime. The rms electron-bunch durations measured as a function of charge for several initial laser transverse size on the photocathode are reported in Fig. 13. As expected for a space-chargedriven expansion, the bunch duration increases for a smaller initial spot size due to the higher initial charge density.

\section{Transverse emittance}

Finally, the transverse emittances of the produced bunches were measured for different laser transverse spot sizes $\sigma_{c}$ and charges $Q$. The multislit mask located at X3 was used to sample the incoming beam into the emittance dominated beamlet and the resulting distribution at X6 was analyzed. The best emittance measured was $1.7 \pm 0.2 \mu \mathrm{m}$ obtained for the minimum charge $Q=266 \pm 25 \mathrm{pC}$ and $\sigma_{c}=1.2 \pm 0.1 \mathrm{~mm}$. This emittance was minimized by a proper choice of the solenoidal lenses setting; see Fig. 14. Although this value is well above state-of-the-art achievements, it represents a $\sim 50 \%$ improvement compared to the typical emittances measured for similar charge at the A0 photoinjector [45].

As pointed out in Ref. [8], the blow-out regime does not a fortiori provide the best transverse emittance. In fact a simple argument based on Eq. (1) and assuming a perfect emittance compensation process indicates that the best achievable transverse normalized emittance scales approximately as

$$
\varepsilon_{\min } \simeq\left[\frac{Q}{\pi \epsilon_{0} E_{0}} \frac{\delta E_{K}}{12 m c^{2}}\right]^{1 / 2}
$$

where $\delta E_{k}$ is the excess in kinetic energy and $m c^{2}$ the electronic rest energy. The latter equation hints that the blow-out regime is generally unfavorable to emittance when operating with high-charge bunches produced in low-peak-field rf guns. For this reason, directly shaping the laser profile to follow an ellipsoidal distribution would

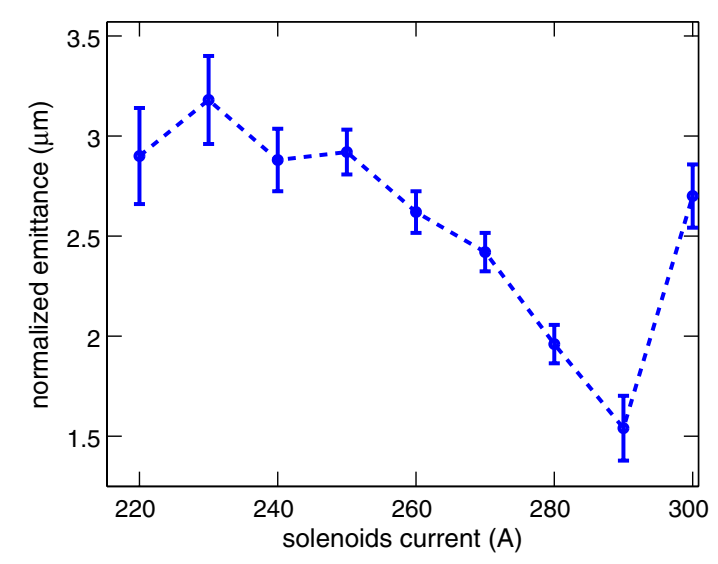

FIG. 14. Normalized transverse horizontal emittance measured as a function of the current setting of the solenoidal lenses (shown as L1, L2, and L3 in Fig. 2) for $Q=266 \pm 25$ pC. be more effective when an improvement of the transverse emittance is desired [5,46] and for our range of bunch charges.

\section{SUMMARY}

We have experimentally demonstrated the production of a uniformly filled ellipsoidal bunch in an L-band rf photoinjector by illuminating a $\mathrm{Cs}_{2} \mathrm{Te}$ photocathode with an ultrashort UV pulse. The bunch ellipsoidal character was shown to be preserved after acceleration to $\sim 14 \mathrm{MeV}$ with charges up to $\sim 0.5 \mathrm{nC}$. The presented work is a significant improvement over a previous attempt to produce ellipsoidal bunches from a $\mathrm{Cs}_{2} \mathrm{Te}$ photocathode in an L-band $\mathrm{rf}$ gun [17]. It also complements the low-charge results obtained for the case of metallic photocathodes in Ref. [14].

Further studies are planned at the High-Brightness Electron-beam Source Laboratory (HBESL) which will be commissioned following the A0PI decommissioning. At HBESL, a 3.9-GHz deflecting cavity located downstream of the rf gun will enable a precise characterization of the longitudinal phase space and possibly the exploration of the response time of $\mathrm{Cs}_{2} \mathrm{Te}$ photocathodes.

Finally, the results presented in this paper support the possible implementation of the blow-out regime at the Advanced Superconducting Test Accelerator (ASTA), currently in construction at Fermilab [47], which will incorporate a $\mathrm{Cs}_{2} \mathrm{Te}$ photocathode. Such an implementation at ASTA would enable the production of high-peak-current (10's kA) electron bunches to be used toward advanced accelerator R\&D.

\section{ACKNOWLEDGMENTS}

We are indebted to R. Montiel, J. Santucci, and B. Tennis for their excellent operational and technical supports. We thank M. Church, H. Edwards, E. Harms, A. H. Lumpkin, and V. Shiltsev for their interest and encouragement. One of us (P. P.) wishes to thank Dr. Smith of AS-Photonics and Dr. Gilevich of SLAC for their help with SNLO. This work was supported by DOE Award No. DE-FG02-08ER41532 and DOD DURIP Award No. N00014-08-1-1064 to Northern Illinois University and by the DOE Contract No. DE-AC02-07CH11359 to the Fermi Research Alliance LLC.

[1] O.D. Kellogg, Foundations of Potential Theory (The Murray Printing Company, Cambridge, MA, 1929).

[2] I. M. Kapchinskii and V. V. Vladimirskii, in Proceedings of the International Conference on High Energy Accelerators, CERN, Geneva (Scientific Information Service CERN, Geneva, 1959), p. 274.

[3] G. R. Neil et al., Phys. Rev. Lett. 84, 662 (2000).

[4] C. Limborg-Deprey and P. R. Bolton, Nucl. Instrum. Methods Phys. Res., Sect. A 557, 106 (2006). 
[5] Y. Li and J. Lewellen, Phys. Rev. Lett. 100, 074801 (2008).

[6] L. Serafini, in Advanced Accelerator Concepts edited by J. S. Wurtele, AIP Conf. Proc. No. 413 (AIP, New York, 1993), p. 645.

[7] O. J. Luiten, S. B. van der Geer, M. J. de Loos, F. B. Kiewiet, and M.J. van der Wiel, Phys. Rev. Lett. 93, 094802 (2004).

[8] Y. Li, arXiv:0809.1582.

[9] P. Musumeci, J. T. Moody, and C.M. Scoby, Ultramicroscopy 108, 1450 (2008).

[10] T. van Oudheusden, P. L.E. M. Pasmans, S. B. van der Geer, M. J. de Loos, M. J. van der Wiel, and O. J. Luiten, Phys. Rev. Lett. 105, 264801 (2010).

[11] F. Sannibale, D. Filippetto, and C.F. Papadopoulos, J. Mod. Opt. 58, 1419 (2011).

[12] D. C. Nguyen et al., Nucl. Instrum. Methods Phys. Res., Sect. A 528, 71 (2004).

[13] T. van Oudheusden, Ph.D. dissertation, Eindhoven University of Technology, 2010.

[14] P. Musumeci, J. T. Moody, R. J. England, J. B. Rosenzweig, and T. Tran, Phys. Rev. Lett. 100, 244801 (2008).

[15] J. T. Moody, P. Musumeci, M.S. Gutierrez, J.B. Rosenzweig, and C.M. Scoby, Phys. Rev. ST Accel. Beams 12, 070704 (2009).

[16] J. B. Rosenzweig, A. M. Cook, R. J. England, M. Dunning, S. G. Anderson, and M. Ferrario, Nucl. Instrum. Methods Phys. Res., Sect. A 557, 87 (2006).

[17] B. O’Shea, J. B. Rosenzweig, G. Asova, J. Bähr, M. Hänel, Y. Ivanisenko, M. Khojoyan, M. Krasilnikov, L. Staykov, and F. Stephan, Phys. Rev. ST Accel. Beams 14, 012801 (2011).

[18] The recent study reported in Ref. [17] does not use a short laser pulse and cannot explore the possible limitations coming from the $\mathrm{Cs}_{2} \mathrm{Te}$ response time. In addition, the reported experimental results (e.g., Fig. 6 of Ref. [17]) do not provide any evidence that the conditions for the blowout regime were actually attained: as noted by the authors, the image charge strongly affected the ellipsoidal selfshaping process.

[19] R. Legg, W. Graves, T. Grimm, and P. Piot, in Proceedings of the 11th European Particle Accelerator Conference, Genoa, 2008 (EPS-AG, Genoa, Italy, 2008), p. 469.

[20] J. Bisognano, M. Bissen, R. Bosch, M. Green, K. Jacobs, H. Hoechst, K. Kleman, R. Legg, R. Reininger, R. Wehlitz, W. Graves, F. Kaertner, and D. Moncton, in Proceedings of the 2009 Particle Accelerator Conference, Vancouver, BC, Canada (TRIUMF, Vancouver, 2010), MO4PBC04.

[21] J.-P. Carneiro, N. Barov, H. Edwards, M. Fitch, W. Hartung, K. Floettmann, S. Schreiber, and M. Ferrario, Phys. Rev. ST Accel. Beams 8, 040101 (2005).

[22] S. H. Kong, J. Kinross-Wright, D. C. Nguyen, and R. L. Shieffield, J. Appl. Phys. 77, 6031 (1995).

[23] A. di Bona, F. Sabary, S. Valeri, P. Michelato, D. Sertore, and G. Suberlucq, J. Appl. Phys. 80, 3024 (1996).
[24] B. Aune et al., Phys. Rev. ST Accel. Beams 3, 092001 (2000).

[25] A. H. Lumpkin, A. S. Johnson, J. Ruan, J. Santucci, Y.-E. Sun, R. Thurman-Keup, and H. Edwards, Phys. Rev. ST Accel. Beams 14, 060704 (2011).

[26] J. Li, R. Tikhoplav, and A. Melissinos, Nucl. Instrum. Methods Phys. Res., Sect. A 564, 57 (2006).

[27] T. J. Maxwell, J. Ruan, P. Piot, and A. H. Lumpkin, JINST 7, T11001 (2012).

[28] P. Tournois, Opt. Commun. 140, 245 (1997).

[29] Dazzler pulse shaper system from Fastlite, France.

[30] D. C. Edelstein, E. S. Wachman, L. K. Cheng, W. R. Bosenberg, and C. L. Tang, Appl. Phys. Lett. 52, 2211 (1988).

[31] SNLO is free software developed by Dr. Arlee Smith and available at http://www.as-photonics.com/SNLO.html.

[32] P. P. Ho and R. R. Alfano, Phys. Rev. A 20, 2170 (1979).

[33] H.-S. Albrecht, P. Heist, J. Kleinschmidt, D. V. Lap, and T. Schröder, Appl. Phys. B 55, 362 (1992).

[34] D. J. Kane and R. Trebino, Opt. Lett. 18, 823 (1993).

[35] K. Michelmann, T. Feurer, B. Fernsler, and R. Sauerbrey, Appl. Phys. B 63, 485 (1996).

[36] K. Flöttmann, ASTRA: A space charge algorithm, User's Manual, available from the World Wide Web at http:// www.desy.de/ mpyflo/Astra_dokumentation/ (unpublished).

[37] P. Hartmann, J. Bermuth, D. v. Harrach, J. Hoffmann, S. Kobis, E. Reichert, K. Aulenbacher, J. Schuler, and M. Steigerwald, J. Appl. Phys. 86, 2245 (1999).

[38] I. V. Bazarov, B. M. Dunham, Y. Li, X. Liu, D. G. Ouzounov, C. K. Sinclair, F. Hannon, and T. Miyajima, J. Appl. Phys. 103, 054901 (2008).

[39] G. Ferrini, P. Michelato, and F. Parmigiani, Solid State Commun. 106, 21 (1998).

[40] W. E. Spicer, Phys. Rev. 112, 114 (1958).

[41] M.J. de Loos, S. B. van der Geer, Y.M. Saveliev, V. M. Pavlov, A. J. W. Reitsma, S. M. Wiggins, J. Rodier, T. Garvey, and D. A. Jaroszynski, Phys. Rev. ST Accel. Beams 9, 084201 (2006).

[42] W.E. King, G. H. Campbell, A. Frank, B. Reed, J. F. Schmerge, B. J. Siwick, B. C. Stuart, and P. M. Weber, J. Appl. Phys. 97, 111101 (2005).

[43] D. X. Wang, G. A. Krafft, and C. K. Sinclair, Phys. Rev. E 57, 2283 (1998).

[44] T. Shaftan, J. Wu, W. Graves, H. Loos, A. Doyuran, L. H. Yu, E. D. Johnson, S. Krinsky, J. Rose, and B. Sheehy, in Proceedings of the 2002 European Particle Accelerator Conference (EPAC 2002), Paris, France (EPS-IGA and CERN, Geneva, 2002), p. 834.

[45] J. Ruan, A. S. Johnson, A. H. Lumpkin, R. Thurman-Keup, H. Edwards, R. P. Fliller, T. W. Koeth, and Y.-E. Sun, Phys. Rev. Lett. 106, 244801 (2011).

[46] Y. Li, S. Chemerisov, and J. Lewellen, Phys. Rev. ST Accel. Beams 12, 020702 (2009).

[47] M. Church, S. Nagaitsev, and P. Piot, in Proceedings of the 2007 Particle Accelerator Conference (PAC07), Albuquerque NM (IEEE, New York, 2007), p. 2942. 